\title{
Nonthermal Supermassive Dark Matter
}

\author{
Daniel J. H. Chung, ${ }^{1,2, *}$ Edward W. Kolb, ${ }^{2,3, \dagger}$ and Antonio Riotto ${ }^{4,+, 8}$ \\ ${ }^{1}$ Department of Physics and Enrico Fermi Institute, The University of Chicago, Chicago, Illinois 60637-1433 \\ ${ }^{2}$ NASA/Fermilab Astrophysics Center, Fermilab National Accelerator Laboratory, Batavia, Illinois 60510-0500 \\ ${ }^{3}$ Department of Astronomy and Astrophysics and Enrico Fermi Institute, The University of Chicago, Chicago, Illinois 60637-1433 \\ ${ }^{4}$ Theory Division, CERN, CH-1211 Geneva 23, Switzerland
}

(Received 29 May 1998)

\begin{abstract}
We discuss several cosmological production mechanisms for nonthermal supermassive dark matter and argue that dark matter may be elementary particles of mass much greater than the weak scale. Searches for dark matter should not be limited to weakly interacting particles with mass of the order of the weak scale, but should extend into the supermassive range as well. [S0031-9007(98)07529-2]
\end{abstract}

PACS numbers: 95.35. $+\mathrm{d}, 98.80 . \mathrm{Cq}$

There is conclusive evidence that the dominant component of the matter density in the Universe is dark. The most striking indication of the existence of dark matter $(\mathrm{DM})$ is the observations of flat rotation curves for spiral galaxies [1], indicating that DM in galactic halos is about 10 times more abundant than the luminous component. Dynamical evidence for DM in clusters of galaxies is also compelling. In terms of the critical density, $\rho_{C}=$ $3 H_{0}^{2} M_{\mathrm{Pl}}^{2} / 8 \pi$ with $H_{0} \equiv 100 h \mathrm{~km} \mathrm{sec}^{-1} \mathrm{Mpc}^{-1}$ and $M_{\mathrm{Pl}}$ the Planck mass, the amount of DM inferred from dynamics of clusters of galaxies is $\Omega_{\mathrm{DM}} \equiv \rho_{\mathrm{DM}} / \rho_{C} \gtrsim 0.3$. In addition, the most natural inflation models predict a flat universe, i.e., $\Omega_{0}=1$, while standard big-bang nucleosynthesis implies that ordinary baryonic matter can contribute at most $10 \%$ to $\Omega_{0}$. This means that about $90 \%$ of the matter in our Universe may be dark.

It is usually assumed that DM consists of a species of a new, yet undiscovered, massive particle we denote as $X$. It is also often assumed that the DM is a thermal relic, i.e., it was in chemical equilibrium in the early Universe.

The simple assumption that the DM is a thermal relic is surprisingly restrictive. The limit $\Omega_{X} \lesssim 1$ implies that the mass of a DM relic must be less than about $500 \mathrm{TeV}$ [2]. This upper bound turns out to be fatal to the proposal that DM consists of charged massive particles (CHAMPs, $C^{ \pm}$) [3]. The present experimental limits on superheavy hydrogen and $C^{-} p$ atoms are compatible with the CHAMP scenario only if they are more massive than about $10^{3} \mathrm{TeV}$ [4]. Similarly, current limits from underground detectors exclude the possibility that halo DM consists of colored particles of mass less than $500 \mathrm{TeV}$. The standard lore is that the hunt for DM should concentrate on particles with a mass of the order of the weak scale and with interaction with ordinary matter on the scale of the weak force. This has been the driving force behind the vast effort in DM detectors.

In view of the unitarity argument, in order to consider thermal supermassive dark matter, one must invoke, for example, late-time entropy production to dilute the abundance of these supermassive particles [5], rendering the scenario unattractive. [In this paper, supermassive implies much more massive than the weak scale (about $100 \mathrm{GeV})$.]

In this Letter we argue that recent developments in understanding how matter is created in the early Universe suggests the possibility that DM in the Universe might be naturally composed of nonthermal supermassive states. The supermassive dark matter (SDM) particles $X$ may have a mass possibly as large as the grand unified theory (GUT) scale. We suggest a number of cosmological production mechanisms for nonthermal supermassive dark matter. It is very intriguing that our considerations resurrect the possibility that the dark matter might be charged or even strongly interacting.

We discuss four production mechanisms. We first propose production during reheating of the Universe after inflation. We point out that, if the reheat temperature is denoted as $T_{\mathrm{RH}}$, the present abundance of SDM is proportional to $\left(2000 M_{X} / T_{\mathrm{RH}}\right)^{-7}$, rather than $\exp \left(-M_{X} / T_{\mathrm{RH}}\right)$ as one might naively expect. We then suggest the possibility of SDM production in preheating, making use of previous work that considered production of massive particles for baryogenesis. We then review the possibility of gravitational production of SDM at the end of the inflationary era. Finally, we propose that SDM might be created in the collisions of vacuum bubbles in a first-order phase transition.

There are two necessary conditions for an SDM scenario. First, the SDM must be stable or at least have a lifetime greater than the age of the Universe. This may result from, for instance, supersymmetric theories where the breaking of supersymmetry is communicated to ordinary particles via the usual gauge forces [6]. In particular, the secluded and the messenger sectors often have accidental symmetries analogous to the baryon number. This means that the lightest particle in those sectors might be stable and very massive if supersymmetry is broken at a large scale [7]. Other natural candidates for supermassive DM arise in theories with discrete gauge symmetries [8] and in string theory and $M$ theory [9]. In the $M$-theory 
case, stable or metastable bound states of matter in the hidden sector, called cryptons, seem to be favored over other possible candidates in string or $M$ theory, such as the Kaluza-Klein states associated with extra dimensions. A specific string model that predicts cryptons as hiddensector bound states weighing about $10^{12} \mathrm{GeV}$ is exhibited in [9].

The second condition for SDM is that the particle must not have been in equilibrium when it froze out (i.e., it is not a thermal relic), otherwise $\Omega_{X}$ would be larger than 1 . (In this paper $\Omega_{i}$ refers to the present value for species i.) A sufficient condition for nonequilibrium is that the annihilation rate (per particle) must be smaller than the expansion rate $n \sigma|v|<H$, where $n$ is the number density, $\sigma|v|$ is the annihilation rate times the Møller flux factor, and $H$ is the expansion rate. Conversely, if the SDM was created at some temperature $T_{*}$ and $\Omega_{X}<1$, then it is easy to show that it could not have attained equilibrium. To see this, assume $X$ 's were created in a radiation-dominated universe at temperature $T_{*}$. Then $\Omega_{X}$ is given by $\Omega_{X}=\Omega_{\gamma}\left(T_{*} / T_{0}\right) m_{X} n_{X}\left(T_{*}\right) / \rho_{\gamma}\left(T_{*}\right)$, where $T_{0}$ is the present temperature. (In this paper we will ignore dimensionless factors of order unity.) Using the fact that $\rho_{\gamma}\left(T_{*}\right)=H\left(T_{*}\right) M_{\mathrm{Pl}} T_{*}^{2}$, we find $n_{X}\left(T_{*}\right) / H\left(T_{*}\right)=\left(\Omega_{X} / \Omega_{\gamma}\right) T_{0} M_{\mathrm{Pl}} T_{*} / M_{X}$. Since we may safely take the limit $\sigma|v|<M_{X}^{-2}, n_{X}\left(T_{*}\right) \sigma|v| / H\left(T_{*}\right)$ must be less than $\left(\Omega_{X} / \Omega_{\gamma}\right) T_{0} M_{\mathrm{Pl}} T_{*} / M_{X}^{3}$. Thus, the requirement for nonequilibrium is

$$
\left(\frac{200 \mathrm{TeV}}{M_{X}}\right)^{2}\left(\frac{T_{*}}{M_{X}}\right)<1 .
$$

This implies that, if a nonrelativistic particle with $M_{X} \gtrsim$ $200 \mathrm{TeV}$ was created at $T_{*}<M_{X}$ with a density low enough to result in $\Omega_{X} \leqslant 1$, then its abundance must have been so small that it never attained equilibrium. Therefore, if there is some way to create SDM in the correct abundance to give $\Omega_{X} \sim 1$, nonequilibrium is guaranteed.

An attractive origin for SDM is during the defrosting phase after inflation. It is important to realize that it is not necessary to convert a significant fraction of the available energy into massive particles; in fact, it must be an infinitesimal amount. If a fraction $\epsilon$ of the available energy density is in the form of a massive, stable $X$ particle, then $\Omega_{X}=\epsilon \Omega_{\gamma}\left(T_{\mathrm{RH}} / T_{0}\right)$, where $T_{\mathrm{RH}}$ is the "reheat" temperature. For $\Omega_{X}=1$, this leads to the limit $\epsilon \lesssim 10^{-17}\left(10^{9} \mathrm{GeV} / T_{\mathrm{RH}}\right)$. We will discuss how particles of mass much greater than $T_{\mathrm{RH}}$ may be created after inflation.

In one extreme we might assume that the vacuum energy of inflation is immediately converted to radiation resulting in a reheat temperature $T_{\mathrm{RH}}$. In this case $\Omega_{X}$ can be calculated by integrating the Boltzmann equation with the initial condition $N_{X}=0$ at $T=T_{\mathrm{RH}}$. One expects the $X$ density to be suppressed by $\exp \left(-2 M_{X} / T_{\mathrm{RH}}\right)$; indeed, one finds $\Omega_{X} \sim 1$ for $M_{X} / T_{\mathrm{RH}} \sim 25+0.5 \ln \left(m_{X}^{2}\langle\sigma|v|\rangle\right)$, in agreement with previous estimates [10] that, for $T_{\mathrm{RH}} \sim 10^{9} \mathrm{GeV}$, the SDM mass would be about $2.5 \times 10^{10} \mathrm{GeV}$.

A second (and more plausible) scenario is that reheating is not instantaneous, but is the result of the decay of the inflaton field. In this approach the radiation is produced as the inflaton decays. The SDM density is found by solving the coupled system of equations for the inflaton field energy, the radiation density, and the SDM mass density. The calculation has been recently reported in Ref. [11] with the result $\Omega_{X} \sim m_{X}^{2}\langle\sigma|v|\rangle\left(2000 T_{\mathrm{RH}} / M_{X}\right)^{7}$. Note that the suppression in $M_{X} / T_{\mathrm{RH}}$ is not exponential, but a power law (albeit a large power). Another crucial feature is the rather large factor of 2000. This implies that, for a reheat temperature as low as $10^{9} \mathrm{GeV}$, a particle of mass $10^{12} \mathrm{GeV}$ can be produced in sufficient abundance to give $\Omega_{X} \sim 1$.

The large difference in SDM masses in the two reheating scenarios arises because the peak temperature is much larger in the second scenario, even with identical $T_{\mathrm{RH}}$. Because the temperature decreases as $a^{-3 / 8}$ ( $a$ is the scale factor) during most of the reheating period in the second scenario, it must have once been much greater than $T_{\mathrm{RH}}$. If we assume that the radiation spectrum did not depart grossly from the thermal, the effective temperature having once been larger than $T_{\mathrm{RH}}$ implies that the density of particles with enough energy to create SDM was larger. Denoting as $T_{2}$ the maximum effective temperature for the second scenario, $T_{2} / T_{\mathrm{RH}} \sim\left(M_{\phi} / \Gamma_{\phi}\right)^{1 / 4} \gg 1$, where $\Gamma_{\phi}$ is the effective decay rate of the inflaton. See [11] for details.

Another way to produce SDM after inflation is in a preliminary stage of reheating called "preheating" [12], where nonperturbative quantum effects may lead to an extremely effective dissipational dynamics and explosive particle production. Particles can be created in a broad parametric resonance with a fraction of the energy stored in the form of coherent inflaton oscillations at the end of inflation released after only a dozen oscillation periods. A crucial observation for our discussion is that particles with mass up to $10^{15} \mathrm{GeV}$ may be created during preheating [13-15], and that their distribution is nonthermal. If these particles are stable, they may be good candidates for SDM.

To study how the creation of SDM occurs in preheating, let us take the simplest chaotic inflation potential: $V(\phi)=M_{\phi}^{2} \phi^{2} / 2$ with $M_{\phi} \sim 10^{13} \mathrm{GeV}$. We assume that the interaction term between the SDM and the inflaton field is $g^{2} \phi^{2}|X|^{2}$. Quantum fluctuations of the $X$ field with momentum $\vec{k}$ during preheating approximately obey the Mathieu equation, $X_{k}^{\prime \prime}+[A(k)-2 q \cos 2 z] X_{k}=0$, where $q=g^{2} \phi^{2} / 4 M_{\phi}^{2}, \quad A(k)=\left(k^{2}+M_{X}^{2}\right) / M_{\phi}^{2}+2 q$ (primes denote differentiation with respect to $z=M_{\phi} t$ ). Particle production occurs above the line $A=2 q$ in an instability strip of width scaling as $q^{1 / 2}$ 
for large $q$. The condition for broad resonance, $A-2 q \lesssim q^{1 / 2} \quad[12,13]$, becomes $\left(k^{2}+M_{X}^{2}\right) / M_{\phi}^{2} \lesssim$ $g \bar{\phi} / M_{\phi}$, which yields $E_{X}^{2}=k^{2}+M_{X}^{2} \lesssim g \bar{\phi} M_{\phi}$ for the typical energy of particles produced in preheating. Here $\bar{\phi}$ is the amplitude of the oscillating inflaton field [12]. The resulting estimate for the typical energy of particles at the end of the broad resonance regime for $M_{\phi} \sim 10^{-6} M_{\mathrm{Pl}}$ is $E_{X} \sim 10^{-1} g^{1 / 2} \sqrt{M_{\phi} M_{\mathrm{Pl}}} \sim g^{1 / 2} 10^{15} \mathrm{GeV}$. Supermassive $X$ bosons can be produced by the broad parametric resonance for $E_{X}>M_{X}$, which leads to the estimate that $X$ production will be possible if $M_{X}<g^{1 / 2} 10^{15} \mathrm{GeV}$. For $g^{2} \sim 1$ one would have copious production of $X$ particles as heavy as $10^{15} \mathrm{GeV}$, i.e., 100 times greater than the inflaton mass, which may be many orders of magnitude greater than the reheat temperature. In fact, in an expanding universe $M_{\phi}$ and $\bar{\phi}$ are time-dependent quantities and one should not only have a very large field at the very beginning of the process of preheating, but also have $E_{X}^{2} \lesssim g \bar{\phi} M_{\phi}$ until the end of preheating [16]. These considerations lead to an estimate of the upper bound on $M_{X}$ slightly smaller than $10^{15} \mathrm{~g}^{1 / 2} \mathrm{GeV}$ [16]. Scatterings of $X$ fluctuations off the zero mode of the inflaton field considerably limit the maximum magnitude of $X$ fluctuations to be $\left\langle X^{2}\right\rangle_{\max } \approx M_{\phi}^{2} / g^{2}$ [17]. For example, $\left\langle X^{2}\right\rangle_{\max } \lesssim 10^{-10} M_{\mathrm{Pl}}^{2}$ if $M_{X}=10 M_{\phi}$. This restricts the corresponding number density of created $X$ particles.

For a reheating temperature of the order of $100 \mathrm{GeV}$, the present abundance of SDM with mass $M_{X} \sim 10^{14} \mathrm{GeV}$ is $\Omega_{X} \sim 1$ if $\epsilon \sim 10^{-10}$. This small fraction corresponds to $\left\langle X^{2}\right\rangle \sim 10^{-12} M_{\mathrm{Pl}}^{2}$ at the end of the preheating stage, a value naturally achieved for SDM masses in the GUT range [17]. The creation of SDM through preheating and, therefore, the prediction of the present value of $\Omega_{X}$ are very model dependent. The evolution of the background inflaton field responsible for the $X$ production will be determined by its coupling to other fields since only a negligible fraction of its energy can go into SDM. We feel very encouraged, however, that it is possible to produce supermassive particles during preheating that are as massive as $10^{12} T_{\mathrm{RH}}$.

Another possibility which has been recently investigated is the production of very massive particles by gravitational mechanisms $[18,19]$. In particular, the desired abundance of SDM may be generated during the transition from the inflationary phase to a matter/radiationdominated phase as the result of the expansion of the background spacetime acting on vacuum fluctuations of the dark matter field [18]. A crucial aspect of inflationary scenarios is the generation of density perturbations. A related effect, which does not seem to have attracted much attention, is the possibility of producing matter fields due to the rapid change in the evolution of the scale factor around the end of inflation. Contrary to the first effect, the second one contributes to the homogeneous background energy density that drives the cosmic expansion and is essentially the familiar "particle production" effect of relativistic field theory in external fields.

Very massive particles may be created in a nonthermal state in sufficient abundance for critical density today by classical gravitational effect on the vacuum state at the end of inflation. Mechanically, the particle creation scenario is similar to the inflationary generation of gravitational perturbations that seed the formation of large scale structures. However, the quantum generation of energy density fluctuations from inflation is associated with the inflaton field which dominated the mass density of the Universe and not a generic, subdominant scalar field.

If $0.04 \lesssim M_{X} / H_{e} \lesssim 2$ [18], where $H_{e}$ is the Hubble constant at the end of inflation, DM produced gravitationally can have a density today of the order of the critical density. This result is quite robust with respect to the fine details of the transition between the inflationary phase and the matter-dominated phase. The only requirement is that $\left(H_{e} / 10^{-6} M_{\mathrm{Pl}}\right)^{2}\left(T_{\mathrm{RH}} / 10^{9} \mathrm{GeV}\right) \geqslant 10^{-2}$. The observation of the cosmic background radiation anisotropy does not fix uniquely $H_{e}$, but using $T_{\mathrm{RH}} \lesssim \sqrt{M_{\mathrm{Pl}} H_{e}}$, we find that the mechanism is effective only when $H_{e} \gtrsim 10^{9} \mathrm{GeV}$ (or $M_{X} \gtrsim 10^{8} \mathrm{GeV}$ ).

The distinguishing feature of this mechanism [18] is the capability of generating particles with mass of the order of the inflaton mass even when the SDM only interacts extremely weakly (or not at all) with other particles, including the inflaton. This feature makes the gravitational production mechanism quite model independent and, therefore, more appealing to us than the one occurring at preheating.

Supermassive particles may also be produced in theories where inflation is completed by a first-order phase transition [20]. In these scenarios, the Universe decays from its false vacuum state by bubble nucleation [21]. When bubbles form, the energy of the false vacuum is entirely transformed into potential energy in the bubble walls, but as the bubbles expand, more and more of their energy becomes kinetic and the walls become highly relativistic. Eventually the bubble walls collide.

During collisions, the walls oscillate through each other [22] and the kinetic energy is dispersed into low-energy scalar waves $[22,23]$. If these soft scalar quanta carry quantum numbers associated with some spontaneously broken symmetry, they may even lead to the phenomenon of nonthermal symmetry restoration [24]. We are, however, more interested in the fate of the potential energy of the walls, $M_{P}=4 \pi \eta R^{2}$, where $\eta$ is the energy per unit area of the bubble with a radius of $R$. The bubble walls can be imagined as a coherent state of inflaton particles, so that the typical energy $E$ of the products of their decays is simply the inverse thickness of the wall, $E \sim \Delta^{-1}$. If the bubble walls are highly relativistic when they collide, there is the possibility of quantum production of nonthermal particles with mass well above the mass of 
the inflaton field, up to energy $\Delta^{-1}=\gamma M_{\phi}, \gamma$ being the relativistic Lorentz factor.

Suppose now that the SDM is some fermionic degree of freedom $X$ and that it couples to the inflaton field by the Yukawa coupling $g \phi \bar{X} X$. One can treat $\phi$ (the bubbles or walls) as a classical, external field and the SDM as a quantum field in the presence of this source. We are thus ignoring the backreaction of particle production on the evolution of the walls, but this is certainly a good approximation in our case. The number of SDM particles created in the collisions from the wall's potential energy is $N_{X} \sim f_{X} M_{P} / M_{X}$, where $f_{X}$ parametrizes the fraction of the primary decay products that are supermassive DM particles. The fraction $f_{X}$ will depend in general on the masses and the couplings of a particular theory in question. For the Yukawa coupling $g$, it is $f_{X} \simeq g^{2} \ln \left(\gamma M_{\phi} / 2 M_{X}\right)$ $[23,25]$. Supermassive particles in bubble collisions are produced out of equilibrium and they never attain chemical equilibrium. Assuming $T_{\mathrm{RH}} \simeq 100 \mathrm{GeV}$, the present abundance of SDM is $\Omega_{X} \sim 1$ if $g \sim 10^{-5} \alpha^{1 / 2}$. Here $\alpha^{-1} \ll 1$ denotes the fraction of the bubble energy at nucleation which has remained in the form of potential energy at the time of collision. This simple analysis indicates that the correct magnitude for the abundance of $X$ particles may be naturally obtained in the process of reheating in theories where inflation is terminated by bubble nucleation.

In conclusion, we have suggested that DM may be elementary supermassive dark matter. Its mass may greatly exceed the electroweak scale, perhaps as the GUT scale. This is possible because the SDM was created in a nonthermal state and has never reached chemical equilibrium, thus avoiding the unitarity upper bound of about $10^{2} \mathrm{TeV}$. We have reviewed a number of ways SDM may be created. If reheating after inflation is preceded by a preheating stage, it is certainly possible to produce by resonance effects copious amounts of dark matter particles much heavier than the inflaton mass. We have also argued that the same may occur if inflation is completed by a first-order phase transition. These two scenarios are based on several assumptions about the structure of the theory, coupling constants, and the reheating temperature, but it is comfortable that the desirable abundance of nonthermal massive relics may be generated. Nonthermal SDM may be also created gravitationally at the end of inflation, with a significant mass range for which the SDM particles will have critical density today regardless of the fine details of the inflation matter/radiation transition. This production mechanism involves the dynamics between the classical gravitational field and a quantum field; it needs no fine-tuning of field couplings or any coupling to the inflaton field. We are excited that the recent developments in understanding how matter is created in the early Universe suggests that DM might be supermassive and resurrect the possibility that it might be charged or even strongly interacting. The implications of these fascinating options will be discussed elsewhere [26].

D. J. H. C. and E. W. K. were supported by the DOE and NASA under Grant No. NAG5-7092.

*Email address: djchung@ theory.uchicago.edu

†Email address: rocky@rigoletto.fnal.gov

‡Email address: riotto@nxth04.cern.ch

${ }^{\S}$ On leave from Department of Theoretical Physics, University of Oxford, Oxford, UK.

[1] See, for instance, G. Jungman, M. Kamionkowski, and K. Greist, Phys. Rep. 267, 196 (1995).

[2] K. Griest and M. Kamionkowski, Phys. Rev. Lett. 64, 615 (1990).

[3] A. de Rújula, S.L. Glashow, and U. Sarid, Nucl. Phys. B333, 173 (1990).

[4] J. L. Basdevant et al., Phys. Lett. B 234, 395 (1990).

[5] J. Ellis, J. L. Lopez, and D. V. Nanopoulos, Phys. Lett. B 247, 257 (1990).

[6] See, for instance, G. F. Giudice and R. Rattazzi, hep-ph/ 9801271.

[7] S. Raby, Phys. Rev. D 56, 2852 (1997); T. Han, T. Yanagida, and R. J. Zhang, hep-ph/9804228.

[8] K. Hamaguchi, Y. Nomura, and T. Yanagida, hep-ph/ 9805346.

[9] K. Benakli, J. Ellis, and D. V. Nanopoulos, hep-ph/ 9803333.

[10] V.A. Kuzmin and V. A. Rubakov, astro-ph/9709187.

[11] D. J. Chung, E. W. Kolb, and A. Riotto, "Production of Massive Dark Matter During Reheating" (to be published).

[12] L. A. Kofman, A. D. Linde, and A. A. Starobinsky, Phys. Rev. Lett. 73, 3195 (1994).

[13] E. W. Kolb, A. D. Linde, and A. Riotto, Phys. Rev. Lett. 77, 4290 (1996).

[14] B. R. Greene, T. Prokopec, and T. G. Roos, Phys. Rev. D 56, 6484 (1997).

[15] E. W. Kolb, A. Riotto, and I. I. Tkachev, Phys. Lett. B 424, 348 (1998).

[16] L. A. Kofman, A. D. Linde, and A. A. Starobinsky, Phys. Rev. D 56, 3258 (1997).

[17] S. Khlebnikov and I. I. Tkachev, Phys. Rev. Lett. 79, 1607 (1997).

[18] D. J. Chung, E. W. Kolb, and A. Riotto, hep-ph/9802238.

[19] V. Kuzmin and I. I. Tkachev, hep-ph/9802304.

[20] D. La and P. J. Steinhardt, Phys. Rev. Lett. 62, 376 (1989).

[21] A. H. Guth, Phys. Rev. D 23, 347 (1981).

[22] S. W. Hawking, I. G. Moss, and J. M. Stewart, Phys. Rev. D 26, 2681 (1982).

[23] R. Watkins and L. Widrow, Nucl. Phys. B374, 446 (1992).

[24] E. W. Kolb and A. Riotto, Phys. Rev. D 55, 3313 (1997); E. W. Kolb, A. Riotto, and I. I. Tkachev, Phys. Rev. D 56, 6133 (1997).

[25] A. Masiero and A. Riotto, Phys. Lett. B 289, 73 (1992).

[26] A. de Rújula, G. F. Giudice, E. W. Kolb, and A. Riotto (to be published). 